\title{
Las energías renovables en el ámbito internacional*
}

\author{
Francisco Javier André \\ Luis Miguel de Castro \\ Emilio Cerdá \\ Universidad Complutense de Madrid
}

\section{Resumen}

En este artículo se analiza la evolución de las energías renovables y la situación actual de las mismas a nivel internacional. Considerando el nivel mundial y, más específicamente la Unión Europea, se estudia la evolución del mix energético, tomando un horizonte temporal de 40 años para el primero y de 10 años para el segundo. Posteriormente se analiza la situación de los diferentes recursos renovables, tanto a nivel mundial como en la Unión Europea. Además, se considera la situación de las energías renovables en cinco países concretos, así como la visión de tres importantes instituciones internacionales.

Palabras clave: energía renovable, mix energético, recursos renovables.

Clasificación JEL: Q40, Q42, Q54.

\begin{abstract}
In this paper the evolution of renewable energy and the present day situation at an international level is analyzed. Looking at the world and, more specifically, the European Union level, we study the evolution of the energy mix, taking the timescale of the last 40 years for the former and the last 10 years for the latter. Subsequently we analyze the general situation of the different renewable energy sources, both at world and European Union level. Moreover we look at the situation of renewable energy in five specific countries and how all of the above is seen by three important international institutions.
\end{abstract}

Key words: renewable energy, energy mix, renewable resources.

JEL classification: $Q 40, Q 42, Q 54$.

\section{Introducción}

A principios del siglo XIX el 95 por 100 de la energía primaria que se consumía en el mundo procedía de fuentes renovables. Un siglo después tal porcentaje era del 38 por 100, y a principios del presente siglo era sólo del 16 por 100 (Fouquet, 2009). Sin embargo, la tendencia parece estar cambiando, ya que en muchos países industrializados la proporción de energías renovables ha crecido de manera considerable en las dos últimas décadas. La inversión total en el mundo en energías renovables, que en el

* F. J. André y E. Cerdá agradecen la financiación del proyecto del Ministerio de Ciencia e Innovación y FEDER ECO2009-14586-C2-01. Francisco J. André también agradece la financiación de los proyectos de la Junta de Andaluciá SEJ 04992 y P07-SEJ-02936. 
año 2004 fue de 22.000 millones de dólares USA, ha crecido de manera espectacular, pasando a 130 en 2008, 160 en 2009 y 211 en 2010. Aproximadamente la mitad de los $194 \mathrm{GW}$ estimados de nueva capacidad eléctrica añadidos en el mundo en 2010 corresponde a energías renovables. A principios de 2011 al menos 118 países tenían políticas de apoyo a las energías renovables o algún tipo de objetivo o cuota a nivel nacional, muy por encima de los 55 países que los tenían en 2005 (REN21, 2011).

Las energías renovables han sustituido parcialmente a los combustibles fósiles y a la energía nuclear en cuatro mercados distintos: generación de electricidad, aplicaciones térmicas (calor para procesos industriales, calefacción, refrigeración y producción de agua caliente en el sector doméstico), carburantes para transporte y servicios energéticos sin conexión a red en el ámbito rural en los países en vías de desarrollo.

El creciente interés por las energías renovables se debe a que estas fuentes energéticas contribuyen a la reducción de emisiones de gases de efecto invernadero, así como las emisiones de otros contaminantes locales, permiten disminuir la dependencia energética y contribuyen a la creación de empleo y al desarrollo tecnológico. Estos argumentos a favor de las energías renovables han sido refrendados por numerosos análisis de expertos y son compartidos actualmente por importantes instituciones internacionales, como se discutirá en la última parte de este trabajo.

El objetivo de este artículo consiste en presentar una visión general de la evolución de las energías renovables en los últimos años y de su situación actual a nivel internacional, tanto en el mundo de manera global como en la Unión Europea (UE) en particular, además de revisar algunas experiencias de particular interés a nivel nacional. En el apartado 2 se estudia la evolución que las distintas fuentes energéticas han experimentado en el mundo en los últimos 40 años. El apartado 3 trata sobre dicha evolución en la UE en la última década. En el apartado 4 se analiza la situación de cada uno de los recursos renovables en el mundo, tanto a nivel global como por regiones geográficas y el apartado 5 particulariza este análisis en la UE. El apartado 6 se centra en cinco países concretos: Estados Unidos, China, India, Nueva Zelanda y Dinamarca. El apartado 7 presenta la visión que se tiene de las energías renovables en tres instituciones internacionales: la Agencia Internacional de Energía, el Panel Intergubernamental para el Cambio Climático y la Agencia Internacional para la Energía Renovable. Finalmente en el apartado 8 se presentan las conclusiones más importantes del trabajo.

\section{La evolución de las fuentes de energía en el mundo en las últimas décadas}

La estructura de la energía primaria por tipos de fuentes es muy distinta según se analiza la situación del mundo en su conjunto o sólo en los países más desarrollados económicamente. El Cuadro 1 permite realizar dicha comparación con los datos más actuales disponibles, así como la evolución del peso de las distintas fuentes energéticas desde el año 1973. 
En el Cuadro 1 se puede observar cómo tras los combustibles fósiles (petróleo, carbón y gas natural) el siguiente grupo en importancia es el que la Agencia Internacional de la Energía (IEA: International Energy Agency) denomina «biomasa y residuos», que comprende biomasa sólida, biomasa líquida, biogás, residuos industriales y residuos urbanos.

\section{CUADRO 1}

ESTRUCTURA DE ENERGÍA PRIMARIA (En porcentajes)

\begin{tabular}{|l|c|c|c|c|}
\hline \multicolumn{1}{|c|}{ Tipo de combustible } & Mundo, 1973 & Mundo, 2009 & OCDE, 1973 & OCDE, 2010 \\
\hline Petróleo & 46,0 & 32,8 & 52,6 & 36,3 \\
\hline Carbón & 24,6 & 27,2 & 22,6 & 20,2 \\
\hline Gas natural & 16,0 & 20,9 & 18,9 & 24,5 \\
\hline Biomasa y residuos & 10,6 & 10,2 & 2,3 & 4,7 \\
\hline Nuclear & 0,9 & 5,8 & 1,3 & 11,0 \\
\hline Hidroeléctrica & 1,8 & 2,3 & 2,1 & 2,1 \\
\hline Geotérmica/solar/eólica & 0,1 & 0,8 & 0,2 & 1,2 \\
\hline Total & 100 & 100 & 100 & 100 \\
\hline En Mtep & 6.111 & 12.150 & 3.741 & 5.413 \\
\hline
\end{tabular}

FUENTE: IEA (2011c).

Comparando los datos de energía primaria en todo el mundo entre 1973 y 2009 se observa que:

- El suministro total de energía prácticamente se ha duplicado entre 1973 y 2009 , pasando de 6.111 a 12.150 Mtep (millones de toneladas equivalentes de petróleo).

- Se ha reducido de manera significativa la aportación del petróleo, pasando de un 46 por 100 en 1973 al 32,8 por 100 en 2009.

- El peso del grupo biomasa y residuos desciende muy ligeramente.

- Aumenta la aportación de la energía nuclear, el gas natural, el carbón, el grupo geotérmica/solar/eólica y la hidroeléctrica.

En el caso de los países que forman la OCDE, se observa que el suministro total de energía se ha incrementado un 38,2 por 100 en el período analizado, pasando de 3.741 Mtep en 1973 a 5.170 Mtep en 2009, bastante menos que a nivel mundial. En el grupo de biomasa y residuos, se observa, por un lado, que en la OCDE el porcentaje es bastante menor que en el mundo en su conjunto y, por otro, que a diferencia de lo que ocurre a nivel mundial, en la OCDE el peso de estos combustibles ha crecido pasando del 2,3 por 100 en 1973 al 4,7 por 100 en 2010 .

Según el Informe sobre el Desarrollo Mundial 2010, del Banco Mundial, titulado Desarrollo y Cambio Climático (Banco Internacional de Reconstrucción y Fomento/ 
Banco Mundial, 2010), en el año 2006, el porcentaje de la biomasa y los residuos sobre el total del suministro de energía primaria estuvo por encima del 50 por 100 en 26 países y por encima del 75 por 100 en los 11 países siguientes: Haití (75,8 por 100), Sudán (77,5 por 100), Zambia (78,2 por 100), Camerún (79,2 por 100), Nigeria (79,6 por 100), Mozambique (81,6 por 100$)$, Togo (84,5 por 100), Nepal $(86,2$ por 100), Etiopía (90 por 100), Tanzania (91 por 100) y República Democrática del Congo $(92,4$ por 100). Tal porcentaje fue del 3,8 por 100 en los países de la OCDE y del 5 por 100 en la Unión Europea-15. Si agrupamos los países por niveles de ingresos, se obtiene un 3,4 por 100 para los países de ingreso alto, 12,3 por 100 para países de ingreso medio y 53,8 por 100 para los países de ingreso bajo.

Hay que distinguir entre usos tradicionales y usos modernos de la biomasa. Los usos tradicionales se identifican principalmente, aunque no exclusivamente, con la biomasa que se obtiene sin mediar una transacción comercial. Se trata mayoritariamente de la leña usada para la cocina y la calefacción. La biomasa moderna se caracteriza por ser objeto de transacciones en el mercado y se utiliza para la generación de energía eléctrica, la producción de calor industrial y doméstico y la producción de biocarburantes para el transporte.

El Banco Mundial está realizando importantes estudios a nivel de país en una línea de trabajo sobre desarrollo bajo en carbono. El primero de dichos estudios se hizo en México (The International Bank for Reconstruction and Development/The World Bank, 2009), y en el mismo se puede apreciar perfectamente la transición desde la biomasa tradicional a la biomasa moderna.

\section{La evolución de las fuentes de energía en la Unión Europea en la última década}

En el Cuadro 2 se presenta la producción de energía primaria en la Unión Europea-27 por fuente energética, entre 1999 y 2009. A la vista de los datos que ofrece dicho cuadro se observa que:

- La producción total de energía primaria en la UE-27 se ha reducido un 15 por 100 entre 1999 y 2009.

- De todas las fuentes de energía, sólo las energías renovables han incrementado su producción (en la UE-27) en la última década, y lo han hecho en un 59 por 100.

- En la UE-27 el petróleo es el combustible que más ha visto disminuir su producción en la última década, con un 42 por 100. Le siguen el carbón (26 por 100) y el gas (25 por 100).

- En 2009, último año para el que se dispone de datos oficiales procedentes de Eurostat, la producción de energía primaria en la UE-27 se reparte de la siguiente forma en cuanto a la fuente utilizada: nuclear (28,8 por 100), carbón $(20,6$ por 100$)$, gas (19 por 100), renovables (18,5 por 100$)$, petróleo (13 por $100)$. 


\section{CUADRO 2}

PRODUCCIÓN ANUAL DE ENERGÍA PRIMARIA POR FUENTE ENERGÉTICA EN LA UE-27 (EN MTEP) (Entre 1999 y 2009)

\begin{tabular}{|l|r|r|c|c|c|c|c|c|c|c|c|c|}
\hline & $\mathbf{1 9 9 9}$ & $\mathbf{2 0 0 0}$ & $\mathbf{2 0 0 1}$ & $\mathbf{2 0 0 2}$ & $\mathbf{2 0 0 3}$ & $\mathbf{2 0 0 4}$ & $\mathbf{2 0 0 5}$ & $\mathbf{2 0 0 6}$ & $\mathbf{2 0 0 7}$ & $\mathbf{2 0 0 8}$ & $\mathbf{2 0 0 9}$ & $\begin{array}{c}\text { Cambio } \\
\text { \% }\end{array}$ \\
\hline Petróleo & 180 & 173 & 161 & 166 & 156 & 145 & 133 & 121 & 120 & 112 & 104 & -42 \\
\hline Gas & 203 & 208 & 208 & 204 & 200 & 203 & 189 & 179 & 167 & 168 & 153 & -25 \\
\hline Nuclear & 243 & 244 & 253 & 256 & 257 & 260 & 258 & 255 & 241 & 242 & 231 & -5 \\
\hline Carbón & 224 & 213 & 211 & 210 & 207 & 201 & 195 & 191 & 185 & 177 & 165 & -26 \\
\hline Renovables & 93 & 97 & 100 & 97 & 104 & 111 & 115 & 122 & 133 & 141 & 148 & +59 \\
\hline Total & 943 & 935 & 933 & 933 & 924 & 920 & 890 & 868 & 846 & 840 & 801 & -15 \\
\hline
\end{tabular}

FUENTE: Elaboración propia, a partir de EUROSTAT (2011).

- Las energías renovables han pasado de representar el 9,8 por 100 de la producción de energía primaria en 1999 al 18,5 por 100 en 2009, en la UE-27.

Mientras la producción de energía primaria se ha ido reduciendo en la UE, la dependencia energética ha ido aumentando, como se puede observar en el Cuadro 3.

\section{CUADRO 3}

DEPENDENCIA ENERGÉTICA EN LA UE-27 (Entre 1999 y 2009) (\%)

\begin{tabular}{|l|c|c|c|c|c|c|c|c|c|c|c|}
\hline & $\mathbf{1 9 9 9}$ & $\mathbf{2 0 0 0}$ & $\mathbf{2 0 0 1}$ & $\mathbf{2 0 0 2}$ & $\mathbf{2 0 0 3}$ & $\mathbf{2 0 0 4}$ & $\mathbf{2 0 0 5}$ & $\mathbf{2 0 0 6}$ & $\mathbf{2 0 0 7}$ & $\mathbf{2 0 0 8}$ & $\mathbf{2 0 0 9}$ \\
\hline UE27 & 45,1 & 46,7 & 47,4 & 47,6 & 49,0 & 50,2 & 52,5 & 53,7 & 53,0 & 54,7 & 53,9 \\
\hline
\end{tabular}

FUENTE: EUROSTAT (2011).

En los 27 países de la Unión Europea la dependencia energética ha aumentado un 9 por 100 aproximadamente entre 1999 y 2009. En 2009, los países de la UE con mayor dependencia energética fueron Malta (100 por 100), Luxemburgo (97,6 por 100), Chipre (97,3 por 100$)$, Irlanda ( 88 por 100$)$, Italia ( 82,9 por 100$)$, Portugal ( 80,9 por $100)$, España ( 79,4 por 100$)$, Bélgica $(74,2$ por 100$)$ y Grecia $(67,8$ por 100$)$, siendo los países con menor dependencia energética Reino Unido (26,6 por 100), Estonia $(21,2$ por 100$)$ y Rumanía $(20,3$ por 100$)$. Dinamarca $(-18,8$ por 100$)$ es el único país de la UE que es exportador neto de energía. Por combustibles, el porcentaje de dependencia energética en la UE-27 es del 62,2 por 100 para el carbón, el 64,2 por 100 para el gas natural y el 83,5 por 100 para el petróleo.

Otro dato a tener en cuenta es el de la intensidad energética, que ha mejorado de manera significativa en la UE-27 entre 1999 y 2009, como se pude apreciar en el Cuadro 4. 


\section{CUADRO 4}

INTENSIDAD ENERGÉTICA EN LA UE-27 (Entre 1999 y 2009)

\begin{tabular}{|l|c|c|c|c|c|c|c|c|c|c|c|}
\hline & $\mathbf{1 9 9 9}$ & $\mathbf{2 0 0 0}$ & $\mathbf{2 0 0 1}$ & $\mathbf{2 0 0 2}$ & $\mathbf{2 0 0 3}$ & $\mathbf{2 0 0 4}$ & $\mathbf{2 0 0 5}$ & $\mathbf{2 0 0 6}$ & $\mathbf{2 0 0 7}$ & $\mathbf{2 0 0 8}$ & $\mathbf{2 0 0 9}$ \\
\hline Kgep/1000 euros & 193 & 187 & 188 & 185 & 187 & 184 & 181 & 175 & 169 & 167 & 165 \\
\hline Índice $(2000=100)$ & 103,0 & 100,0 & 100,2 & 98,7 & 99,7 & 98,3 & 96,6 & 93,7 & 90,1 & 89,4 & 88,2 \\
\hline
\end{tabular}

FUENTE: EUROSTAT (2011).

En el año 2009, los países de la UE-27 con mejores resultados en cuanto a intensidad energética fueron Dinamarca (107 Kgep/1000 euros), Irlanda (109) y Reino Unido (114), y los peores Rumanía (577), Estonia (607) y Bulgaria (843). España (168) ocupaba el lugar 11 entre los países con menos intensidad energética.

La elevada dependencia energética de la UE se debe que su consumo de energía es muy superior a su producción. El Cuadro 5 muestra los datos del consumo interior bruto anual por fuente energética. Se observa que:

- El consumo de energía primaria en la UE-27 creció hasta 2006 y descendió desde 2007, de modo que el consumo total en 2009 es similar al del año 1999.

- Por fuentes energéticas, sólo ha aumentado el consumo de gas natural (en un 9 por 100) y, sobre todo, el de energías renovables (en un 65 por 100). Se ha reducido el consumo de la energía nuclear (en un 5 por 100), el petróleo (7 por 100) y el carbón (14 por 100).

- En el año 2009, el consumo de energía primaria en la UE-27 se reparte de la siguiente forma, en cuanto a la fuente utilizada: petróleo (36,8 por 100), gas natural $(24,7$ por 100$)$, carbón $(15,8$ por 100$)$, nuclear $(13,7$ por 100$)$, energías renovables ( 9 por 100).

- Las energías renovables han pasado de representar el 5,5 por 100 del consumo de energía primaria en 1999 al 9 por 100 en 2009 en el conjunto de 27 países de la UE.

\section{CUADRO 5}

CONSUMO INTERIOR BRUTO POR FUENTE ENERGÉTICA EN LA UE-27 (EN MTEP), ANUAL (Entre 1999 y 2009)

\begin{tabular}{|l|r|r|r|r|r|r|r|r|r|r|r|c|}
\hline & $\mathbf{1 9 9 9}$ & $\mathbf{2 0 0 0}$ & $\mathbf{2 0 0 1}$ & $\mathbf{2 0 0 2}$ & $\mathbf{2 0 0 3}$ & $\mathbf{2 0 0 4}$ & $\mathbf{2 0 0 5}$ & $\mathbf{2 0 0 6}$ & $\mathbf{2 0 0 7}$ & $\mathbf{2 0 0 8}$ & $\mathbf{2 0 0 9}$ & $\begin{array}{c}\text { Cambio } \\
\%\end{array}$ \\
\hline Petróleo & 671 & 661 & 676 & 671 & 675 & 677 & 678 & 674 & 659 & 658 & 623 & -7 \\
\hline Gas & 383 & 394 & 404 & 405 & 425 & 435 & 446 & 438 & 433 & 441 & 417 & 9 \\
\hline Nuclear & 243 & 244 & 253 & 256 & 257 & 260 & 258 & 255 & 241 & 242 & 231 & -5 \\
\hline Carbón & 313 & 321 & 323 & 319 & 331 & 327 & 318 & 325 & 328 & 306 & 268 & -14 \\
\hline Renovables & 93 & 97 & 100 & 98 & 104 & 112 & 116 & 124 & 135 & 144 & 153 & +65 \\
\hline Total & 1.703 & 1.717 & 1.756 & 1.749 & 1.792 & 1.811 & 1.816 & 1.816 & 1.796 & 1.791 & 1.692 & $-0,65$ \\
\hline
\end{tabular}

FUENTE: Elaboración propia, a partir de EUROSTAT (2011). 


\section{Situación general de las energías renovables en el mundo}

De acuerdo con la IEA, en 2009 la oferta total de energía primaria en el mundo fue de 12.169 Mtep, de la cual el 13,1 por 100 fue producida a partir de fuentes renovables. En el Cuadro 6 aparecen los porcentajes correspondientes a cada fuente energética renovable.

\begin{tabular}{|c|c|}
\hline $\begin{array}{r}\text { CUAD } \\
\text { PORCENTAJE DE CADA RECURSO } \\
\text { TOTAL DE ENERGÍA PRIMARIA REI }\end{array}$ & $\begin{array}{l}\text { SOBRE LA OFERTA } \\
\text { EL MUNDO (EN 2009) }\end{array}$ \\
\hline Recurso renovable & Porcentaje \\
\hline Biomasa sólida & 70,2 \\
\hline Hidráulica & 17,7 \\
\hline Geotérmica & 3,9 \\
\hline Biocarburantes & 3,4 \\
\hline Eólica & 1,5 \\
\hline Biogas & 1,4 \\
\hline Solar y mareas & 1 \\
\hline Fracción orgánica de residuos sólidos urbanos & 0,9 \\
\hline
\end{tabular}

FUENTE: IEA (2001d).

Debido al amplio uso de la biomasa tradicional de tipo no comercial (para cocinar y calentar las viviendas), en los países en vías de desarrollo la biomasa sólida es, con mucha diferencia, el recurso renovable más utilizado, representando el 9,2 por 100 de la oferta de energía primaria total (OEPT) en el mundo y el 70,2 por 100 de la oferta de energía renovable global. La energía hidráulica ocupa la segunda posición, con el 2,3 por 100 de la OEPT en el mundo, el 17,7 por 100 en el ámbito de las energías renovables. La energía geotérmica supone el 0,5 por 100 de la OEPT y el 3,9 por 100 de las renovables. Los biocarburantes le siguen de cerca, con el 0,4 por 100 de la OEPT y el 3,4 por 100 de las renovables. Entre la eólica, la solar y energía mareomotriz cubren el 0,3 por 100 de la OEPT, o el 2,5 por 100 de las renovables.

Desde 1990 las energías renovables han crecido a una tasa media anual de 1,8 por 100 , ligeramente más que la oferta total de energía primaria (1,7 por 100). El Gráfico 1 muestra las tasas medias de crecimiento anual para las distintas fuentes renovables. Se observa un elevado crecimiento de la solar fotovoltaica y la eólica, que partían de valores muy bajos en 1990 . 


\section{GRÁFICO 1}

TASAS MEDIAS DE CRECIMIENTO ANUAL DE LA OFERTA DE ENERGÍAS RENOVABLES EN EL MUNDO DESDE 1990 (hasta 2009)



FUENTE: IEA (2001d).

En el Cuadro 7 se presenta la contribución de las energías renovables a la oferta de energía primaria total en 2009 para diferentes zonas geográficas. La biomasa tradicional tiene un peso muy importante en países en vías de desarrollo, especialmente en el sur de Asia y en el África subsahariana. De hecho, el 86 por 100 de la energía obtenida a partir de biomasa sólida se produce en países que no forman parte de la OCDE, donde también se produce el 59,7 por 100 de la energía hidráulica mundial. En cambio, las «nuevas renovables» como la solar, eólica, mareas, biogás, biocarburantes y fracción orgánica de residuos sólidos urbanos (FORSU) son producidas en países de la OCDE en un 70,4 por $100^{1}$.

En cuanto a la energía eléctrica, la generación de electricidad renovable en el mundo ha crecido desde 1990 a una tasa media anual del 2,8 por 100, de modo similar a la generación total de electricidad. En dicha generación, la contribución de las fuentes renovables en su conjunto ocupó en 2009 el tercer lugar, con una contribución del 19,3 por 100 , por detrás del carbón $(40,4$ por 100$)$ y del gas $(21,4$ por 100$)$, pero por delante de la energía nuclear (13,4 por 100) y del petróleo (5,2 por 100). Entre 1990 y 1999 el peso de la electricidad renovable ha disminuido ligeramente de un 19,5 por 100 a un 19,3 por 100, debido a que, en este periodo, la producción de energía hidroeléctrica bajó del 18,2 por 100 al 16,3 por 100. En dichos años, la participación del resto de las renovables aumentó desde un 1,3 por 100 hasta un 3,0 por 100. En la OCDE, la electricidad renovable ha crecido desde 1990 a una tasa media anual del 1,7 por 100. En los países no pertenecientes a la OCDE la electricidad renovable creció al 4 por 100 frente a un 4,5 por 100 de crecimiento de la electricidad en su conjunto.

\footnotetext{
${ }^{1}$ La biomasa (sólida) moderna también se considera como «nueva renovable»
} 


\section{CUADRO 7}

CONTRIBUCIÓN DE LAS ENERGÍAS RENOVABLES A LA OFERTA DE ENERGÍA PRIMARIA TOTAL (OEPT), POR REGIONES, EN EL MUNDO (en 2009)

\begin{tabular}{|l|r|c|c|c|c|c|}
\hline & OEPT & $\begin{array}{c}\text { De la cual, } \\
\text { renovables }\end{array}$ & $\begin{array}{c}\text { Aportación } \\
\text { renovables } \\
\text { en OEPT }\end{array}$ & \multicolumn{3}{|c|}{$\begin{array}{c}\text { Aportación de las principales } \\
\text { categorías en el total de renovables } \\
\text { \%) }\end{array}$} \\
\hline & Mtep & Mtep & $\%$ & Hidro & $\begin{array}{c}\text { Solar, eól, } \\
\text { geot, mare }\end{array}$ & $\begin{array}{c}\text { Biomasa y } \\
\text { residuos }\end{array}$ \\
\hline Africa & 663,9 & 321,6 & 48,4 & 2,6 & 0,4 & 97,0 \\
\hline América Latina & 567,9 & 177,1 & 31,2 & 33,7 & 1,9 & 64,4 \\
\hline Asia (sin China) & 1449,8 & 388,6 & 26,8 & 5,4 & 6,9 & 87,7 \\
\hline China & 2272,0 & 267,9 & 11,8 & 19,8 & 4,2 & 76,0 \\
\hline No OCDE Europa y Eurasia & 1038,0 & 39,5 & 3,8 & 63,6 & 1,6 & 34,7 \\
\hline Oriente Medio & 610,7 & 3,0 & 0,5 & 36,9 & 39,7 & 23,5 \\
\hline OCDE & 5237,7 & 391,5 & 7,5 & 29,0 & 14,5 & 56,5 \\
\hline Transp. marítimo y aéreo int. & 328,9 & $\mathrm{x}$ & $\mathrm{x}$ & $\mathrm{x}$ & $\mathrm{x}$ & $\mathrm{x}$ \\
\hline Mundo & $12.169,0$ & $1.589,3$ & 13,1 & 17,7 & 6,4 & 75,9 \\
\hline
\end{tabular}

FUENTE: IEA (2011d).

Respecto a la participación de las distintas fuentes renovables, la hidráulica aporta el 84 por 100 de la electricidad renovable, seguida de la biomasa y los residuos con un 6,7 por 100 . El resto (9,3 por 100) corresponde a la energía eólica, solar y geotérmica, que están creciendo rápidamente.

En los Cuadros 8 y 9 se presentan ordenados los cinco países líderes a nivel mundial en cuanto a recursos añadidos y capacidad instalada, respectivamente, con datos de 2010 .

\section{CUADRO 8}

CINCO PAÍSES LÍDERES MUNDIALES EN CUANTO A RECURSOS AÑADIDOS (en 2010)

\begin{tabular}{|c|c|c|c|c|c|c|}
\hline & $\begin{array}{c}\text { Inversión en } \\
\text { capacidad } \\
\text { nueva }\end{array}$ & $\begin{array}{c}\text { Energía } \\
\text { eólica }\end{array}$ & $\begin{array}{c}\text { Energía } \\
\text { solar } \\
\text { fotovoltaica }\end{array}$ & $\begin{array}{c}\text { Energía } \\
\text { solar para } \\
\text { calor y agua } \\
\text { caliente }\end{array}$ & $\begin{array}{c}\text { Producción } \\
\text { de etanol }\end{array}$ & $\begin{array}{c}\text { Producción } \\
\text { de biodiesel }\end{array}$ \\
\hline $\mathbf{1}$ & China & China & Alemania & China & EEUU & Alemania \\
\hline $\mathbf{2}$ & Alemania & EEUU & Italia & Alemania & Brasil & Brasil \\
\hline $\mathbf{3}$ & EEUU & India & Rep. Checa & Turquía & China & Argentina \\
\hline $\mathbf{4}$ & Italia & España & Japón & India & Canadá & Francia \\
\hline $\mathbf{5}$ & Brasil & Alemania & EEUU & Australia & Francia & EEUU \\
\hline
\end{tabular}

FUENTE: REN21(2001). 


\section{CUADRO 9}

CINCO PAÍSES LÍDERES MUNDIALES EN CUANTO A CAPACIDAD

INSTALADA (A finales de 2010)

\begin{tabular}{|c|c|c|c|c|c|c|c|}
\hline & $\begin{array}{c}\text { Capacidad } \\
\text { instalada } \\
\text { en reno- } \\
\text { vables } \\
\text { (sin hidro.) }\end{array}$ & $\begin{array}{c}\text { Capacidad } \\
\text { instalada } \\
\text { en reno- } \\
\text { vables } \\
\text { (con hidro. }\end{array}$ & $\begin{array}{c}\text { Capacidad } \\
\text { instalada } \\
\text { energía } \\
\text { eléc. } \\
\text { eólica }\end{array}$ & $\begin{array}{c}\text { Capacidad } \\
\text { instalada } \\
\text { energía } \\
\text { eléc. } \\
\text { biomasa }\end{array}$ & $\begin{array}{c}\text { Capacidad } \\
\text { instalada } \\
\text { energía } \\
\text { eléc. geo- } \\
\text { térmica }\end{array}$ & $\begin{array}{c}\text { Solar } \\
\text { foto- } \\
\text { voltaica }\end{array}$ & $\begin{array}{c}\text { Energía } \\
\text { solar para } \\
\text { calor y } \\
\text { agua } \\
\text { caliente }\end{array}$ \\
\hline $\mathbf{1}$ & EEUU & China & China & EEUU & EEUU & Alemania & China \\
\hline $\mathbf{2}$ & China & EEUU & EEUU & Brasil & Filipinas & España & Turquía \\
\hline $\mathbf{3}$ & Alemania & Canadá & Alemania & Alemania & Indonesia & Japón & Alemania \\
\hline $\mathbf{4}$ & España & Brasil & España & China & México & Italia & Japón \\
\hline $\mathbf{5}$ & India & $\begin{array}{c}\text { Alemania/ } \\
\text { India }\end{array}$ & India & Suecia & Italia & EEUU & Grecia \\
\hline
\end{tabular}

FUENTE: REN21 (2011).

\section{Situación general de las energías renovables en la Unión Europea}

Según los últimos datos facilitados por el consorcio Eurobserv'ER, referidos a la UE-27 para el año 2010 se pueden obtener las siguientes conclusiones:

- La cuota de energías renovables del consumo energético final bruto fue del 12,4 por 100 en 2010 (11,5 por 100 en 2009).

- La cuota de energías renovables del consumo eléctrico total fue del 19,8 por 100 en 2010 (18,2 por 100 en 2009).

- La cuota de energías renovables del consumo energético interno bruto fue del 9,9 por 100 en 2010 (9,1 por 100 en 2009).

- El empleo basado en las energías renovables fue de 1,14 millones en $2010(0,91$ millones en 2009).

- La actividad económica basada en las energías renovables fue de 127 millones de euros en 2010 (120 millones de euros en 2009).

En el año 2010 el consumo de energía primaria renovable en la UE-27 fue de 172,5 Mtep (un 11,3 por 100 más que en 2009). En el Cuadro 10 se presenta el porcentaje de cada fuente sobre el total de renovables. 


\section{CUADRO 10}

PORCENTAJE DE CADA RECURSO RENOVABLE EN LA CUOTA DE ENERGÍAS RENOVABLES EN EL CONSUMO DE ENERGÍA PRIMARIA EN LA UE-27 (en 2010)

\begin{tabular}{|l|l|}
\hline Bioenergía & 68,2 por 100 \\
\hline Hidráulica & 18 por 100 \\
\hline Eólica & 7,4 por 100 \\
\hline Geotérmica & 4,4 por 100 \\
\hline Solar & 2 por 100 \\
\hline Energías marinas & 0,03 por 100 \\
\hline
\end{tabular}

FUENTE: EurObserv'ER (2011).

La bioenergía, tal como aparece recogido en el Cuadro 10, se refiere al conjunto de biomasa sólida, biogás, biocarburantes y FORSU, con los siguientes porcentajes (dentro del conjunto del recurso bioenergía):

- Biomasa sólida: 70,9 por 100.

- Biocarburantes: 12,4 por 100 .

- Biogás: 9,7 por 100 .

- FORSU: 7,1 por 100 .

Los países de la UE con mayor porcentaje de energías renovables en su consumo de energía primaria en el año 2010 han sido Suecia (35,9 por 100), Letonia $(35,4$ por 100$)$, Austria (27,6 por 100), Finlandia (26,6 por 100$)$, Portugal (22,2 por 100) y Dinamarca (20,3 por 100). España (11,3 por 100) ocupaba el undécimo lugar.

La producción de electricidad renovable en la UE-27 en 2010 fue de 661,4 TWh (un 12,75 por 100 más que en 2009). El Cuadro 11 muestra el porcentaje de cada recurso renovable dentro del 19,8 por 100 de cuota de las renovables en el consumo eléctrico total.

\section{CUADRO 11}

PORCENTAJE DE CADA RECURSO RENOVABLE EN LA CUOTA DE ENERGÍAS RENOVABLES EN EL CONSUMO ELÉCTRICO TOTAL EN LA UE-27 (en 2010)

\begin{tabular}{|l|c|}
\hline Hidráulica & 54,5 por 100 \\
\hline Eólica & 22,5 por 100 \\
\hline Bioenergía & 18,6 por 100 \\
\hline Solar & 3,5 por 100 \\
\hline Geotérmica & 0,8 por 100 \\
\hline Energías marinas & 0,1 por 100 \\
\hline
\end{tabular}

FUENTE: EurObserv'ER (2011). 
Dentro del 18,6 por 100 correspondiente al conjunto de la bioenergía, la biomasa sólida representa el 59,5 por 100, el biogás el 25,8 por 100 y la FORSU el 14,7 por 100.

Los países de la UE con mayor porcentaje de energías renovables en su consumo eléctrico total en el año 2010 han sido Austria (60,6 por 100), Suecia (54,7 por 100), Portugal (50,9 por 100), Letonia (48,4 por 100$)$, Eslovenia ( 34,4 por 100$)$, Dinamarca $(33,3$ por 100$)$ y España $(33,1$ por 100$)$.

2010 era un año de referencia importante para las energías renovables en la UE, ya que para este año se habían establecido objetivos indicativos en el Libro Blanco y las directivas 2001/77/EC y 2003/30/EC. En particular, el Libro Blanco sobre fuentes renovables de energía, publicado por la Comisión Europea en noviembre de 1997, fija como objetivo indicativo una aportación del 12 por 100 de las energías renovables en el consumo de energía primaria en el año 2010, frente al 6 por 100 que había en ese momento. En el Anexo 1 del Libro Blanco se proponen las medidas estratégicas concretas y en su Anexo 2 se especifican las contribuciones estimadas (indicativas) para cada fuente renovable. Según EurObserv'ER, en 2010 la aportación de las energías renovables en el consumo de energía primaria ha sido del 10,2 por 100 (incluyendo la producción de energía hidroeléctrica bombeada), por lo que se ha llegado al 85 por 100 del objetivo del 12 por 100 que se fijó en 1997.

En 2001 se adoptó la Directiva 2001/77/EC del Parlamento Europeo y del Consejo sobre promoción de electricidad renovable. Esta directiva establece como objetivo incrementar la participación de las renovables en la generación de electricidad, hasta un 22 por 100 del consumo eléctrico bruto en 2010 (partiendo de una participación del 14 por 100). El objetivo del 22 por 100 para la UE-15 pasó a ser del 21 por 100 para la UE-25 y luego para la UE-27. Se estableció para los países miembros la obligación de fijar sus propios objetivos para un horizonte temporal de 10 años, teniendo en cuenta el objetivo europeo y los compromisos nacionales adquiridos en el Protocolo de Kioto. Según EurObserv'ER la participación de las energías renovables en la generación de electricidad ha sido del 19,8 por 100 , con lo que se ha conseguido el 94,3 por 100 del objetivo que se estableció en la Directiva. Entre los 27 paises de la UE, los que han cumplido con los objetivos que tenían marcados en este aspecto han sido Bélgica, República Checa, Dinamarca, Estonia, Alemania, Hungría, Lituania, Holanda, Portugal, Eslovenia, Irlanda y España.

La Directiva 2003/30/EC del Parlamento Europeo y del Consejo sobre promoción del uso de biocarburantes y otros carburantes renovables para el transporte en sustitución de gasolina y diesel pretende contribuir a los objetivos de cumplimiento de los compromisos en materia de cambio climático, seguridad de suministro con respeto al medio ambiente y promoción de las energías renovables. Esta Directiva establece un objetivo indicativo para la UE del 5,75 por 100 de sustitución de carburantes convencionales para el transporte por biocarburantes en diciembre de 2010 . Se ha conseguido un nivel del 4,7 por 100 , por lo que se ha alcanzado el 82 por 100 del objetivo que se marcó en 2003. 
Por otra parte, 2010 fue el año que marcó el primer aniversario de la adopción de la Directiva de energías renovables (2009/28/EC) sobre promoción del uso de energía producida a partir de fuentes renovables, que establece un objetivo obligatorio del 20 por 100 de cuota de energías renovables en el consumo de energía final para 2020 (el objetivo del 20 por 100 es para el conjunto de la UE, teniendo cada país un objetivo particular). En el Cuadro 12 se presenta para cada uno de los 27 países de la UE el porcentaje de energías renovables sobre el consumo bruto de energía final en 2010 y el objetivo obligatorio para 2020.

\section{CUADRO 12}

PORCENTAJE DE ENERGÍAS RENOVABLES EN EL CONSUMO FINAL BRUTO EN 2010 Y OBJETIVOS NACIONALES PARA 2020 DE LA DIRECTIVA 2009/28/EC

\begin{tabular}{|c|c|c|c|c|c|c|c|c|c|c|}
\hline País & Suecia & Letonia & $\begin{array}{c}\text { Finlan- } \\
\text { dia }\end{array}$ & Austria & Portugal & Estonia & $\begin{array}{c}\text { Dina- } \\
\text { marca }\end{array}$ & $\begin{array}{c}\text { Eslo- } \\
\text { venia }\end{array}$ & $\begin{array}{c}\text { Ruma- } \\
\text { nía }\end{array}$ & $\begin{array}{c}\text { Litua- } \\
\text { nia }\end{array}$ \\
\hline $\mathbf{2 0 1 0}$ & 46,9 & 34,3 & 33,6 & 30,7 & 24,7 & 24,1 & 23 & 21,7 & 21,4 & 21,1 \\
\hline ON2020 & 49 & 40 & 38 & 34 & 31 & 25 & 30 & 25 & 24 & 23 \\
\hline País & España & Bulgaria & Francia & $\begin{array}{c}\text { Eslova- } \\
\text { quia }\end{array}$ & $\begin{array}{c}\text { Alema- } \\
\text { nia }\end{array}$ & Polonia & R. Checa & Grecia & Italia & Hungría \\
\hline $\mathbf{2 0 1 0}$ & 14,1 & 12,9 & 12,4 & 11,4 & 10,7 & 9,9 & 9,7 & 9,1 & 8,5 & 8,5 \\
\hline ON2020 & 20 & 16 & 23 & 14 & 18 & 15 & 13 & 18 & 17 & 13 \\
\hline País & Irlanda & Chipre & Bélgica & Holanda & R. Unido & Luxemb. & Malta & UE-27 & & \\
\hline $\mathbf{2 0 1 0}$ & 5,9 & 5,5 & 5,4 & 3,8 & 3,3 & 2,6 & 0,3 & $\mathbf{1 2 , 4}$ & & \\
\hline ON2020 & 16 & 13 & 13 & 13 & 15 & 11 & 10 & $\mathbf{2 0}$ & & \\
\hline
\end{tabular}

FUENTE: EurObserv'ER (2011).

\section{Situación de las energías renovables en algunos países significativos}

En esta sección se resumen algunas experiencias nacionales especialmente interesantes, ya sea por el tamaño y la relevancia de los países implicados (como los casos de Estados Unidos, China e India) o por la existencia de compromisos particularmente claros (como los casos de Nueva Zelanda y Dinamarca).

\section{Estados Unidos}

Los datos para 2010 (Center for Sustainable Systems, University of Michigan, 2011) indican que el 83 por 100 de la energía final consumida en USA proviene de combustibles fósiles (petróleo, gas natural y carbón), un 9 por 100 de la energía nuclear y un 8 por 100 de energías renovables. De éstas, la más importante es la bio- 
masa (53 por 100 del total) seguida de la hidroeléctrica (31 por 100) y la eólica (12 por 100). Pero estas cifras están sumamente alejadas de los potenciales estimados. El Departamento de Energía estimó en 2008 que el potencial de la energía eólica en USA alcanzaba un 20 por 100 del total del consumo eléctrico. Y el laboratorio nacional de energías renovables estimaba en 2004 que si se dedicara un 0,4 por 100 de la superficie a la producción de energía solar fotovoltaica, se podría cubrir la totalidad del consumo de energía eléctrica del país.

Entre las renovables, el mayor crecimiento lo está experimentando la energía eólica (15 por 100 en 2010) cuya capacidad instalada alcanza $40.2 \mathrm{GW}$, solo por detrás de China $(44.7 \mathrm{GW})$ y supone el 0,9 por 100 del total de la energía usada en el país.

En la primera década de este siglo los precios de la gasolina se incrementaron en USA, desde $1,5 \$$ por galón en 2001 a $2 \$$ por galón en 2005 y a cerca de $3 \$$ por galón en 2007. Esta situación propició iniciativas legislativas como la Ley de Política Energética de 2005, la Ley de Independencia y Seguridad Energética de 2007 y la Ley de Extensión y Mejora Energética de 2008. Pero estos cambios legislativos buscaban la seguridad del suministro y la reducción de los niveles de dependencia y no se planteaba la necesidad de cambiar el modelo energético. Solo en la ley de 2007 se recogía el reconocimiento del Congreso de que las energías renovables debían contribuir al menos en un 25 por 100 del consumo total de energía (Isbell 2010). La contribución de las energías renovables ha ido aumentando de forma continuada desde los años 50, con el 3 por 100 del consumo total, hasta alcanzar un 6 por 100 en el año 2000. (Center for Sustainable Systems. University of Michigan, 2011). Entre los años 1990 y 2009 la contribución de energías renovables al total de la oferta de energía primaria ha pasado del 5 al 5,6 por 100 (IEA 2011d).

La administración Obama inició su andadura con un impulso decidido hacia las energías renovables, consiguiendo un avance que no se había producido desde el final de la década de los años 70. En Noviembre de 2009 se produjo la asociación con China en materia de energías renovables, eficiencia energética y tecnologías bajas en emisiones de $\mathrm{CO}_{2}$. $\mathrm{Al}$ mismo tiempo se creó el nuevo Consejo para la Energía entre la Unión Europea y USA. Ese mismo año se promulgó la Ley Americana de Energía Limpia y de Seguridad que estableció, para el año 2020, un objetivo de reducción del 17 por 100 con respecto a las emisiones de gases de efecto invernadero de 2005 .

\section{China}

Las profundas transformaciones que se han producido en China en los últimos cincuenta años hacen que los consumos energéticos en este país sean muy dispares. El 60 por 100 de su población vive en áreas rurales donde el consumo de energía final procede de biomasa tradicional en un 74 por 100 y el consumo eléctrico está basado en pequeñas estaciones hidroeléctricas (Ma et al., 2009). En las áreas urbanas y en las instalaciones industriales los combustibles fósiles son los únicos significativos, con un gran predominio del carbón como fuente de energía eléctrica. En 
términos de producción energética el carbón ha pasado de suponer el 72,8 por 100 en 1985 al 76,7 por 100 en 2006, mientras que la participación del petróleo era del 20,9 por 100 en 1985 y bajó hasta el 11,9 por 100 en 2006. En términos de consumo, las cifras son 75,8 y 69,4 para el carbón y 17,1 y 20,4 para el petróleo.

China ha experimentado un crecimiento económico espectacular en las últimas tres décadas lo cual ha significado un aumento del consumo energético y de los niveles de contaminación, que sitúan a China como uno de los actores principales en la lucha contra el cambio climático. Los incrementos en el consumo de energía primaria fueron del 15 por 100 en 2003 y del 16 por 100 en 2004. Los últimos datos referidos a 2010 muestran un crecimiento del 9,6 por 100, que está por encima de lo planificado. Esto significa más del 20 por 100 del consumo energético mundial (Yuan y otros, 2011). En 2006, las emisiones chinas sobrepasaban a las de Estados Unidos, según la agencia ambiental de los Países Bajos (Wang y otros, 2010), y sus emisiones suponen ya un 25 por 100 del total mundial.

El crecimiento de sus emisiones es muy elevado, debido a que su principal fuente de producción de energía es el carbón. ${ }^{2}$ Al mismo tiempo, su producción de energías renovables es casi insignificante en términos relativos, a pesar de su fuerte incremento en los últimos años, que hace que, en términos absolutos, China se sitúe ya en los primeros lugares del mundo en algunas de las energías renovables. En 2010, las energías no fósiles suponen un 8,3 por 100 del total de la energía primaria consumida.

Los datos referidos a 2010 sobre generación de energías indicaban que la hidroeléctrica significaba un 22,2 por 100 , la eólica un 3,2 por 100, la energía nuclear el 1,1 por 100 y las energías térmicas el 73,4 por 100 (China Briefing News, junio de 2011).

En cuanto a la capacidad eólica instalada, China fue duplicando su capacidad cada año, entre 2006 y 2008 y sobrepasó a India para alcanzar el primer puesto en Asia. Además, China alcanzó en 2009 la segunda posición mundial junto con Alemania, y en 2010 alcanzó la primera posición superando a USA (Cheung 2011). Pero la situación en términos comparativos es muy distinta. Mientras en Estados Unidos el 45 por 100 de la nueva capacidad instalada en los años 2007 y 2008 corresponde a energía eólica, el dato en China para el mismo periodo de tiempo es del 6 por 100 (Wang et al., 2010).

Aunque China estableció algunos programas de energías renovables en la década de los 90, no es hasta la promulgación de la Ley de Energía Renovable de 2005 cuando podemos hablar de una iniciativa legislativa de alcance capaz de desarrollar en alguna medida la producción y el uso de estas energías. Como consecuencia de esta ley, el Plan de Desarrollo de Energías Renovables correspondiente al undécimo Plan Quinquenal, estableció que el uso de estas energías tenía que alcanzar un 10 por 100 del total en 2010 y un 20 por 100 en 2020 . Y en este mismo plan quinquenal se estableció el objetivo de reducir la intensidad energética en un 20 por 100 para 2010

${ }^{2}$ En 2007 el 93 por 100 de la energía consumida provenía de combustibles fósiles, de los cuales un 75 por 100 era carbón (NBSC 2008). 
en relación a 2005. Esto significó un cambio estratégico en la política energética y una muestra de la importancia dada por el régimen chino a las cuestiones relacionadas con el cambio climático y energía (Yuan et al., 2011).

A finales de 2009 y justo antes de celebrarse la Conferencia sobre Cambio Climático de Copenhague, se anunció un nuevo objetivo de reducción para 2020 de entre un 40 y un 45 por 100 con relación a 2005, incrementando la participación de energías no fósiles hasta un 15 por 100 del total para ese 2020. El duodécimo Plan Quinquenal (2011-2015) persigue una reducción del 17 por 100 en la relación carbón/PIB, una reducción de intensidad energética del 16 por 100 y una participación de energías no fósiles del 11,4 por 100 sobre el total.

La política energética china tiene dos objetivos en relación con la reducción de emisiones. Por un lado, la reducción de la intensidad energética, y por otro, el desarrollo de energías renovables. Pero puesto que China tiene un objetivo de crecimiento económico del 7,2 por 100 anual para el periodo 2000 a 2020 (Yang, 2008), la reducción de combustibles fósiles no parece posible. A pesar de los crecimientos observados entre 2005 y 2007, la participación de energías renovables en el total de la producción eléctrica había decrecido un 1,23 por 100 (Wang, Yin y Li 2010).

El compromiso de China con la conservación de energías y la reducción de emisiones parece establecido y ha dado ya algunos resultados. Desde 1980 hasta 2005, China ha conseguido un 3,9 por 100 anual de ahorro de energía. En 2006 se estableció un objetivo de reducir la relación energía/producto en un 20 por 100 para 2010 en relación con los niveles de 2005, como ya hemos comentado, y a finales de 2010 se había conseguido un 19,1 por 100 de reducción (Yuan et al., 2011).

Las previsiones de la demanda energética en China muestran un crecimiento muy considerable debido a varios factores:

1. El consumo de electricidad per cápita es el 25 por 100 del promedio mundial y por tanto sujeto a enormes aumentos.

2. El fenómeno de la urbanización del país es muy notable. La población urbana ha pasado del 30 por 100 en 1996 hasta el 44 por 100 en 2006. En las áreas urbanas, el consumo eléctrico per cápita es casi el doble que en las zonas rurales.

3. El gran crecimiento de los medios de transporte públicos y privados, que han pasado de 4,18 millones de vehículos en 1995 a 26,2 millones en 2006.

Debido a los enormes crecimientos de su demanda energética, China debe diversificar su oferta, y en este sentido el énfasis está en la energía hidroeléctrica, aunque sin olvidar que tiene un enorme potencial de producción de energía solar, eólica y biomasa. Y a pesar de lo sucedido en Japón, el desarrollo de la energía nuclear puede llegar a jugar un papel importante en los planes a largo plazo. 


\section{India}

Resulta significativo el hecho de que en India existe un ministerio llamado «Ministerio Energías Nuevas y Renovables» (Ministry of New and Renewable Energy) ${ }^{3}$ En un reciente documento de dicho ministerio (Ministry of New and Renewable Energy. Government of India, 2010), se destaca el hecho de que en las últimas décadas, a pesar de la considerable expansión de la capacidad eléctrica instalada, el crecimiento económico y la urbanización han hecho que la demanda crezca más todavía, lo que ha producido apagones y episodios de escasez energética. Esta situación constituye, sin duda, una de las principales causas que han impulsado a India a profundizar en la búsqueda de energías renovables.

Desde el año 2002 la capacidad de la red renovable se ha multiplicado por más de 5, constituyendo en torno al 10,9 por 100 de la capacidad total de la red en el país y contribuyendo alrededor del 4,13 por 100 en el mix de generación de electricidad.

La energía renovable se ha convertido en un importante elemento estratégico para India y el gobierno ha puesto en marcha diversas iniciativas para la promoción de estas fuentes energéticas. La Ley de la Electricidad de 2003 fue el primer marco normativo en que se incorporó el apoyo a las renovables, con tarifas preferentes, obligación de compra de energía renovable, créditos y certificados de energía renovable. Además, el gobierno ha apoyado a la energía renovable mediante diversos incentivos fiscales y financieros que incluyen subsidios, amortización acelerada, exención de derechos de aduana, incentivos basados en la generación o feed-in tariffs.

Aunque todos los sectores renovables están creciendo, es el eólico el que lo está haciendo de un modo más notable, contribuyendo en torno a un 75 por 100 de la capacidad de generación renovable conectada a red. India también es abundante en energía solar, y éste es el recurso que se considerada con el mayor potencial de futuro. La llamada misión solar nacional Jawaharlal Nehru establece para 2022 un objetivo de 20.000 MW de capacidad de generación de energía solar de red, 2.000 MW fuera de red, incluyendo 20 millones de sistemas de iluminación con energía solar y 20 millones de metros cuadrados de colectores térmicos.

Entre las prioridades del gobierno indio, también se incluye incrementar la electrificación rural, para poder satisfacer la demanda no cubierta por medio de energía renovable. En los últimos dos años, se ha suministrado energía a 150 localidades en el estado de Bihar mediante miniredes basadas en gasificación por medio de la cáscara del arroz. Para 2022 se planea abastecer 10.000 localidades mediante sistemas basados en la biomasa y más de 1000 localidades mediante energía solar. Se han instalado plantas de biogás para abastecer de energía para cocinar en las areas rurales a 4,27 millones de familias y para 2022 se planea instalar 2 millones de plantas más.

Mediante el desarrollo de aplicaciones renovables descentralizadas, en cinco años el ministerio de energías renovables pretende ahorrar un millón de litros de

${ }^{3}$ Dicho Ministerio tomó su nombre actual en 2006, sustituyendo al anterior «Ministerio de Fuentes de Energía no Convencionales».. 
combustibles fósiles al año, especialmente gasóleo y keroseno. Este ahorro espera lograrse en gran parte en las torres de telecomunicaciones y los centros de generación energética industriales. Además, el gobierno de India ha decidido que todos sus nuevos edificios deberán adaptarse a los requerimientos para tener, al menos, 3 o 4 estrellas en el sistema nacional indio de edificios sostenibles, GRIHA (Green Rating for Integrated Habitat Assessment).

En un estudio de Ernst and Young se ha situado a India como el cuarto país más atractivo para realizar inversiones en energía renovable, sólo por detrás de Estados Unidos, China y Alemania.

\section{Nueva Zelanda}

En Nueva Zelanda las energías renovables supusieron un 33 por 100 del total de la oferta primaria en 2008. Destacan en especial las hidroeléctricas y geotérmicas. Ese mismo año, el 64 por 100 de la producción eléctrica era procedente de energías renovables y alcanzó el 73 por 100 en 2009. Es el tercer país de la OCDE de mayor contribución, sólo por detrás de Noruega y Austria. Su objetivo es llegar al 90 por 100 en 2025.

Peo tal vez el dato más destacado de Nueva Zelanda es que en 2007 anunció su objetivo de alcanzar la neutralidad en términos de carbono, es decir, alcanzar cero emisiones netas. Para ello el Gobierno tomaría la iniciativa a través del diseño de consumos eficientes en los edificios públicos, la eliminación de despilfarros en el consumo, el aumento del reciclaje, la sustitución de viajes por teleconferencias y el mayor uso de vehículos de poco consumo energético. Y en última instancia las emisiones remanentes deben ser canceladas a través del incremento de masa forestal y la compra de permisos de emisión.

En Julio de 2008 se introdujo el NZETS (Mercado de Derechos de Emisión de Nueva Zelanda) para una serie de sectores y con el objetivo de cubrir la totalidad de los mismos en 2015. En 2010 se publicó el documento de estrategia energética que incluye medidas de eficiencia energética y con unos objetivos de reducción de emisiones del 10 al 20 por 100 entre 1990 y 2020 y del 50 por 100 entre 1990 y 2050.

\section{Dinamarca}

En 2010 Dinamarca ocupó la octava posición entre los miembros de la IEA por su participación de energías renovables, tanto en lo referente a la oferta total de energías primarias $(20,7$ por 100$)$ como en el apartado de producción eléctrica $(33,5$ por 100$)$. En lo que respecta a la oferta total el principal aporte viene dado por la biomasa y los residuos con un 17,1 por 100. En electricidad la energía eólica supone un 20,2 por 100 y la biomasa y los residuos un 13,2 por 100. Hay que tener en cuenta que Dinamarca no posee recursos hidroeléctricos (IEA, 2012). 
Estas cifras suponen un avance muy considerable en la utilización de energías renovables, teniendo en cuenta que en el año 2000, las participaciones eran del 11,4 por 100 en la oferta total y el 17,1 por 100 en lo referente a electricidad. La contribución de energías renovables a la oferta total de energía primaria en Dinamarca ha pasado de una contribución del 6,2 por 100 en 1990 a un 17,4 por 100 en 2009.

Los objetivos que se plantea este país son muy ambiciosos. En 2011 el gobierno de Dinamarca hizo público que pretende prescindir totalmente de los combustibles fósiles para 2050. Es esta la última de una serie de iniciativas que se iniciaron en los años 80 y que han continuado hasta la actualidad. Su objetivo para la participación de renovables en el consumo energético final bruto es del 30 por 100 en 2020.

\section{Visión de las energías renovables desde algunas instituciones internacionales}

En el apartados 5 ha quedado patente la importancia que se concede en la UE a las energías renovables y cómo éstas han ido creciendo a un ritmo importante en los últimos años. Sin embargo, no es la Unión Europea la única institución que ha mostrado un claro posicionamiento a favor de las energías renovables. En este apartado se resume la visión de otras tres importantes instituciones internacionales como son la IEA, el Panel Intergubernamental para el Cambio Climático (IPCC: Intergovernmental Panel on Climate Change) y la Agencia Internacional para la Energía Renovable (IRENA, International Renewable Energy Agency).

\subsection{Agencia Internacional de la Energía (IEA)}

En sus informes de los últimos años, la IEA ha señalado de modo sistemático que la energía renovable ha crecido rápidamente en la última década, convirtiéndose en un importante componente de la oferta energética (véase, como muestra, IEA 2008, 2010, 2011a, 2011b). También señala la IEA que muchos inversores han venido considerando este sector una alternativa atractiva. Un primer motivo para este atractivo tiene que ver con los altos precios de los combustibles fósiles. Un segundo motivo se debe al creciente apoyo de los gobiernos a las renovables. Apoyo que obedece a los esfuerzos por reducir las emisiones de gases de efecto invernadero y al deseo de diversificar la oferta y la seguridad en el suministro, además del positivo impacto de las renovables sobre la creación de empleo. En consecuencia con estos propósitos, cada vez más gobiernos están adoptando objetivos y tomando medidas para aumentar la participación de las renovables en el mix energético. Según la IEA, la base de recursos para las renovables es muy amplia y puede satisfacer una parte considerable de la demanda energética mundial. No obstante, la mayoría de las renovables no son competitivas en las actuales condiciones de mercado y dependen en gran medida de estos incentivos públicos. 
En sus proyecciones a futuro basadas en escenarios alternativos, la IEA predice un considerable aumento de la energía renovable. Este aumento es común a todos los escenarios previstos y también es común a todos ellos el pronóstico de que las ayudas públicas seguirán siendo un factor determinante para este aumento. Se espera que la participación de las renovables no hidráulicas en la generación de energía pase del 3 por 100 en 2009 al 15 por 100 en 2035, gracias a las ayudas públicas, que se multiplicarían por cinco, pasando a unos 180.000 millones de dólares. En su estudio reciente sobre el desarrollo de las renovables (IEA 2011b) la Agencia Internacional de la Energía señala que las tasas de crecimiento observadas de las renovables en los últimos años se corresponden aproximadamente con las tasas requeridas para cumplir las proyecciones de la IEA sobre un futuro energético sostenible.

Según las predicciones de la IEA, los principales actores de la expansión de las renovables serán China y la Unión Europea, responsables de más o menos la mitad del crecimiento total. También se pronostica que, aunque los subsidios por unidad de producción disminuirán, habrá más fuentes renovables necesitadas de ayudas para poder desarrollarse y competir en los mercados eléctricos. La IEA estima que, aunque estas ayudas son costosas financieramente a corto plazo, contribuirán a generar beneficios duraderos a largo plazo en términos de seguridad energética y protección del medio ambiente.

Para progresar en el desarrollo de las renovables, es preciso superar algunas barreras. Algunas de las barreras más evidentes son de tipo económico y están relacionadas fundamentalmente con los costes de producción, transporte y distribución. Sin embargo, en su informe de 2008 acerca del desarrollo de las renovables (IEA 2008), la Agencia Internacional de la Energía también advierte de la presencia de barreras no económicas como las trabas administrativas, las deficiencias en el diseño de los mercados eléctricos, la falta de información y entrenamiento y los problemas de aceptabilidad social.

Para contribuir al desarrollo de las renovables y la integración de las mismas en un futuro energético sostenible, la IEA sugiere unos principios rectores para el diseño de políticas (véase IEA 2011b, p. 21). El primero de estos principios requiere proporcionar un marco predecible y transparente de promoción de las renovables, integrado dentro de una estrategia energética más amplia que se estructure en torno a objetivos ambiciosos y creíbles. El segundo principio consiste en adoptar un enfoque dinámico que tenga en cuenta el estado de madurez de cada tecnología así como las tendencias de los mercados, tanto nacionales como internacionales. El tercer principio aconseja abordar las barreras no económicas de un modo sistemático, diseñando procesos y procedimientos en la medida en que sea posible. El cuarto principio se dirige de modo particular a las fases iniciales de desarrollo y aconseja que, en esta fase, se aborden cuidadosamente los aspectos relacionados con la integración de las energías renovables en el sistema energético, y que podrían conllevar barreras en las fases posteriores, tales como las infraestructuras o el diseño de los mercados. 


\subsection{Panel Intergubernamental para el Cambio Climático (IPCC)}

Recientemente, el IPCC ha publicado un informe con el propósito de evaluar la literatura científica sobre el papel potencial de la energía renovable para la mitigación del cambio climático, analizar los obstáculos para el desarrollo de este tipo de energía y las estrategias para superar dichos obstáculos (véase IPCC, 2011)

Según observa el IPCC, las tecnologías asociadas a la energía renovable se han desarrollado considerablemente en los últimos años y se espera que lo sigan haciendo. El IPCC estima que en 2008 la energía renovable supuso un 12,9 por 100 de la oferta mundial de energía primaria. La fuente renovable que representó un porcentaje más alto (con el 10,2 por 100) fue la biomasa, de la cual, la mayoría (en torno al 60 por 100) fue biomasa tradicional dedicada a la cocina y el calentamiento de los hogares en países en desarrollo, pero también experimentó un notable desarrollo la llamada biomasa moderna. La otra gran fuente de energía a nivel global fue la energía hidroeléctrica, con un 2,3 por 100 y el resto de las fuentes renovables alcanzaron un 0,4 por 100 .

Según el IPPC, el desarrollo de las renovables se explica por factores como el aumento en la demanda global de energía, el encarecimiento de los combustibles fósiles o las políticas aplicadas por diversos países. Pero también tienen mucho que ver con las reducciones en los costes de muchas de las tecnologías renovables, que se han ido materializando en las últimas décadas a pesar de ciertos altibajos en los precios. Se espera que los costes de las renovables sigan bajando más todavía, gracias a diversos factores tales como la posibilidad de emplear nuevos y mejores sistemas de producción y suministro de materias primas, el desarrollo de los biocarburantes de nueva generación, el desarrollo de la tecnología solar térmica y fotovoltaica, las nuevas tecnologías oceánicas, los mejores diseños para las turbinas eólicas offshore y la capacidad de hacer las instalaciones hidroeléctricas rentables en un mayor número de localizaciones.

Al igual que otros estudios, en el informe del IPCC se concluye que, además de poseer un considerable potencial para mitigar el cambio climático, la energía renovable puede proporcionar muchos más beneficios, contribuyendo a un desarrollo social y económicamente sostenible, a facilitar el acceso a la energía y la seguridad del suministro, reducir la volatilidad del mercado energético, crear puestos de trabajo y reducir impactos negativos sobre el medio ambiente y la salud de las personas.

A pesar del desarrollo de las renovables, en la fase actual un crecimiento adecuado de su participación en el mix energético requiere la implantación de políticas para estimular cambios en el sistema energético y atraer las inversiones necesarias en nuevas tecnologías e infraestructuras. Merced a diversos estudios de la literatura, el IPCC concluye que el potencial tecnológico de la energía renovable es bastante mayor que la demanda energética mundial. El mayor potencial técnico corresponde a la energía solar, pero también existe un potencial considerable para el resto de renovables. Incluso en las regiones con bajo potencial técnico, existen notables oportunidades de crecimiento con respecto a los niveles actuales. 


\subsection{Agencia Internacional para la Energía Renovable (IRENA)}

Uno de los hitos recientes más importantes en cuanto al posicionamiento internacional respecto a las energías renovables es la creación de la Agencia Internacional para la Energía Renovable. Ya en la Conferencia de las Naciones Unidas sobre fuentes de energía nuevas y renovables (UN Conference on New and Renewable Sources of Energy), celebrada en Nairobi en 1981 se propuso la creación de una agencia internacional dedicada a la energía renovable. La idea se fue debatiendo y desarrollando en distintas reuniones internacionales en el ámbito de las energías renovables al tiempo que ha ido creciendo el interés por las fuentes energéticas alternativas. Este interés se ha manifestado de modo particular en conferencias como la Cumbre Mundial sobre el Desarrollo Sostenible de Johanesburgo, 2002, o las Conferencias Internacionales sobre Energía Renovable de Bonn, 2004 y Pekín, 2005. En las conclusiones de la Conferencia de Bonn se pedía expresamente la creación de una Agencia Internacional para la Energía Renovable. La primera conferencia preparatoria para la creación de IRENA se celebró en 2008 en Berlín, donde 170 representantes de 60 estados mostraron su apoyo a la idea de que IRENA se crease lo antes posible. Posteriores reuniones de trabajo preparatorias se celebraron en el mismo año en Berlín y Madrid, donde se elaboró el tratado fundacional (el «Estatuto») de IRENA, los mecanismos de financiación, el procedimiento para elegir al Director General, el establecimiento de las oficinas centrales y los planes iniciales de acción.

IRENA fue fundada oficialmente en Bonn, en enero de 2009 y la sede central se ubicó provisionalmente en Abu Dhabi (Emiratos Árabes Unidos). En abril de 2011 se disolvió la Comisión Preparatoria y concluyó el proceso de creación de IRENA. Sus órganos de funcionamiento incluyen la Asamblea (compuesta por todos los miembros de la Agencia), el Consejo (con 21 miembros) y la secretaría. En 2011 se eligió como director general al keniata Adnan Z. Amin. El Estatuto fue firmado inicialmente por 75 estados. Actualmente IRENA cuenta con 89 miembros, incluyendo 88 estados más la Unión Europea, y 68 estados más son aspirantes a serlo. El primer programa de trabajo de la Agencia ha contado con un presupuesto inicial de 25 millones de dólares USA.

IRENA constituye la primera organización de escala global dedicada a la promoción de la energía renovable, lo que supone un importante hito en cuanto al posicionamiento de los gobiernos mundiales frente a las energías alternativas. Según el artículo II del Estatuto, el objetivo de IRENA es el de promover la implantación generalizada y reforzada y el uso sostenible de todas las formas de energía renovable. Los miembros de IRENA se comprometen a promover las energías renovables en sus propias políticas nacionales y a contribuir a la transición hacia un suministro energético seguro y sostenible.

Las actividades previstas de IRENA incluyen fomentar los debates y la interacción con otras organizaciones, difundir información y concienciar a la población mundial acerca de los beneficios y el potencial de las energías renovables. En cuanto a la relación con sus miembros, las tareas de IRENA incluyen analizar, supervisar 
y sistematizar las prácticas en materia de energías renovables, prestar servicios de asesoramiento y apoyar a los miembros en materia de políticas, mejorar los mecanismos de transferencia de conocimientos y tecnología, apoyar a los miembros en la creación de capacidades, entre otras cosas mediante formación y capacitación, facilitar asesoramiento respecto a la financiación de las energías renovables, fomentar la investigación y la creación de redes científicas, la investigación conjunta y el desarrollo e implantación de tecnologías y proporcionar información sobre el desarrollo y aplicación de normas técnicas nacionales e internacionales.

\section{Conclusiones}

El suministro total de energía en el mundo prácticamente se ha duplicado entre 1973 y 2009, reduciéndose de manera significativa el porcentaje de aportación del petróleo y aumentando la contribución de la energía nuclear, el gas natural, el carbón y las energías renovables (en las que la biomasa tradicional tiene todavía una importancia muy destacada). Cuando se analiza dicho período para el conjunto de países que forman la OCDE se observan diferencias considerables respecto al marco global.

En la Unión Europea, en la última década, de todas las fuentes de energía sólo las energías renovables han aumentado su producción, y lo han hecho en un 59 por 100. En cuanto al consumo de energía primaria, sólo han aumentado el gas natural (en un 9 por 100) y, sobre todo, las energías renovables (en un 65 por 100), que han pasado de representar el 5,5 por 100 en 1999 al 9,04 por 100 en 2009.

De acuerdo con la Agencia Internacional de la Energía, en 2009, el 13 por 100 de la oferta total de energía primaria en el mundo fue producida a partir de fuentes renovables. Tal porcentaje fue del 16 por 100, en términos de energía final (REN21, 2011). La biomasa sólida es, con mucha diferencia, el recurso renovable más utilizado en el mundo, seguido de la energía hidroeléctrica y, a una distancia considerable, la geotérmica, los biocarburantes, la energía eólica, el biogás y las energías solares.

La UE lleva años adoptando una postura proactiva a favor de las energías renovables y estableciendo objetivos temporales de exigencia creciente. En particular, en el año 2010, la cuota de energías renovables fue del 9,9 por 100 en el consumo de energía primaria, del 12,4 por 100 en el consumo de energía final y del 19,8 por 100 en el consumo eléctrico, proporcionando 1,14 millones de puestos de trabajo. La bionergía es el recurso renovable que más se utiliza, seguido de la energía hidráulica, la eólica, la geotérmica, la solar y las energías marinas.

De los objetivos indicativos fijados por la UE para el año 2010, el de aportación del 12 por 100 de las energías renovables en el consumo de energía final se ha conseguido en un 85 por 100 , el del 21 por 100 de participación en el consumo eléctrico bruto se ha alcanzado en un 94,3 por 100 , y el del 5,75 por 100 de sustitución de carburantes convencionales para el transporte por biocarburantes se ha conseguido en un 82 por 100. Para el año 2020, se han establecido objetivos obligatorios del 20 
por 100 de renovables en el consumo de energía final y el 10 por 100 en el caso de la energía utilizada para el transporte.

En Estados Unidos, en el año 2010, las energías renovables representaron el 8 por 100 de la energía final consumida y el 10,9 por 100 de la energía primaria de producción doméstica (un 5,6 por 100 más que en 2009), cerca ya del 11,3 por 100 correspondiente a la energía nuclear, siendo la biomasa la más utilizada, seguida de la hidráulica y la eólica (que está experimentando el mayor crecimiento, un 15 por 100 en 2010).

En China, el Plan de Desarrollo de Energías Renovables estableció que el uso de dichas energías tenía que alcanzar un 10 por 100 del total en 2010 y un 20 por 100 en 2020. Actualmente China es el país con mayor capacidad instalada total en energías renovables, y también ocupa este puesto en energía eólica y en energía solar para calefacción y agua caliente. Además, en 2010, China ha sido el país del mundo que más inversión nueva ha realizado en energías renovables.

En India la energía renovable se ha convertido en un importante elemento estratégico y el gobierno ha puesto en marcha distintas iniciativas para la promoción de las mismas. Destaca la energía eólica y el desarrollo de muchas aplicaciones renovables descentralizadas.

En Nueva Zelanda, las energías renovables supusieron en 2008 el 33 por 100 de la oferta primaria total, y en 2009 el 73 por 100 de la producción eléctrica, y el objetivo nacional es llegar al 90 por 100 en 2025. El país anunció en 2007 el objetivo de alcanzar neutralidad en términos de carbono.

Dinamarca tiene como objetivo que la participación de renovables en la oferta total sea del 30 por 100 en 2020. En el año 2011, su gobierno ha anunciado el objetivo de prescindir totalmente de los combustibles fósiles en 2050.

Esta destacada evolución de las energías renovables se justifica por algunas propiedades de las mismas relacionadas con la reducción de emisiones de gases de efecto invernadero y otros contaminantes, la disminución de la dependencia energética, la creación de empleo y el desarrollo tecnológico. Así ha sido contrastado por numerosos estudios científicos y se asume inequívocamente por las principales instituciones internacionales en materia energética. En este sentido, tanto la IEA como el IPCC han elaborado importantes informes sobre la situación y las perspectivas de las energías renovables y han ofrecido recomendaciones para su progresivo desarrollo e implantación. La creación de IRENA, una agencia internacional orientada monográficamente al desarrollo de las energías renovables constituye, un hito significativo dentro de lo que parece un proceso imparable de reforzamiento de dichas energías en el mundo. 


\section{Referencias bibliográficas}

[1] BAnco Internacional de ReConstrucción y Fomento/BAnCo Mundial (2010): Desarrollo y Cambio Climático. Coedición del Banco Mundial, Mundi-Prensa y Mayol Ediciones.

[2] CEnTER FOR Sustainable Systems (University of Michigan) (2011): U.S. Renewable Energy Factsheet. Pub N ${ }^{\mathrm{O}} \mathrm{CSS} 03-12$.

[3] Cheung, K. (2011): Integration of Renewables Status \& Challenges in China. IEA Working Paper.

[4] EUROBSERVER (2011): The State of Renewable Energies in Europe. $11^{\text {th }}$ EurObserv'ER Report. París.

[5] EuROSTAT (2011): Energy, Transport and Environmental Indicators. Eurostat Pocketbooks. European Commission, Luxemburgo.

[6] FouQueT, R. (2009): «A brief history of energy», en J. Evans y L.C. Hunt (eds.), International Handbook of the Economics of Energy, Cheltenham, UK and Northampton, MA, USA: Edward Elgar Publishing, p. 1-19.

[7] IEA (International Energy Agency) (2008): Deploying Renewables. Principles for Effective Policies, OECD/IEA, París.

[8] IEA (International Energy Agency) (2010): World Energy Outlook 2010, OECD/IEA, Paris.

[9] IEA (International Energy Agency) (2011a): World Energy Outlook 2011, OECD/IEA, Paris.

[10] IEA (International Energy Agency) (2011b): Deploying Renewables. Best and Future Policy Practice, OECD/IEA, París.

[11] IEA (Internacional Energy Agency) (2011c): Key World Energy Statistics, OECD/IEA, París.

[12] IEA (International Energy Agency) (2011d): Renewables Information 2011 with 2010 data, OECD/IEA, París.

[13] IEA (International Energy Agency) (2012): Energy Policies of IEA Countries- Denmark- 2011 Review, OECD/IEA, París.

[14] IPCC (2012): Renewable Energy Sources and Climate Change Mitigation. Special Report of the Intergovernmental Panel on Climate Change. Technical Support Unit Working Group III Potsdam Institute for Climate Impact Research (PIK). Cambridge University Press.

[15] ISBELL, P. (2010): «La política energética de la Administración Obama: ¿dónde estamos después del primer año?» Tratado de Energías Renovables, vol. I. Iberdrola, Aranzadi y Thomson Reuters.

[16] MA, H.; OXLEY, L. y GIBSON, J. (2009): «China's energy situation in the new millennium», Renewable and Sustainable Energy Reviews, 13, pp. 1781-1799.

[17] MinisTRY OF NEW AND RENEWABLE ENERGY (Government of India) (2010): Renewable Energy in India: Progress, Vision and Strategy. Disponible en http://www.indiaopportunities.es/documentos/renewable_energy_strategy.da.

[18] NBSC 2008.China Statistics Yearbook of 2008.National Bureau of Statistics of China.

[19] REN21 (2011): Renewables 2011: Global status report. Disponible en www.ren21.net

[20] The InTERnATIONAL BANK For RECONSTRUCTION AND DEVELOPMENT/THE WORLD BANK (2009): Low-Carbon Development for Mexico. Washington DC. 
[21] WANG, F.; YIN, H. y LI, S. (2010): «China's renewable energy policy: Commitments and challenges», Energy Policy, 38, pp. 1872-1878.

[22] YANG, M. (2008): «China’s energy efficiency target», Energy Policy, 36, pp. 561-570.

[23] YUAN, J.; KANG, J.; YU, C. y HU, Z. (2011): «Energy conservation and emissions reduction in China-Progress and prospective«, Renewable and Sustainable Energy Reviews, 15 , pp. 4334-4347. 